\section{Neurophysiological models of animal sentience}

\section{Michele Panzera}

Laboratory of Veterinary Ethology, Department of Veterinary Science, University of Messina, Messina, Italy

\begin{abstract}
The pain in animals is typically inferred on the basis of nonverbal behavior. The proposed model is based on the bioengineering principle that structure determines function. Basic functional homologies can be mapped in the brain regions directly responsible for the sensation of painful stimuli and their representation in the limbic circuits of the sentience.
\end{abstract}

\section{Introduction}

An extensive neurophysiological literature of human pain allows us to believe that it represents the result of neural processing of nociceptive stimuli through specific selected neural cytoarchitectures (i.e. pool or cluster of neurons) and peculiar circuits (connections between these clusters). There are at least three main lines of scientific evidence supporting the cortical origins of human pain: i) brain imaging reveals a dynamic network of cortical activity that provides a neural signature of pain; ii) lesions to cortical regions in this network alter pain sensation and iii) direct stimulation of the cortical regions of this network evokes pain. ${ }^{1}$

\section{Results}

Assuming that the organizational principles are necessary for pain-conscious neural processes, it follows that nociceptive sentience belongs to telencephalic neural structures. Animal taxa lacking any of these neuroanatomical features should not be able of feeling pain. This axiom, however, presents significant inconsistencies, since animal species with different neural cytoarchitectures do not possess neuroanatomy need to feel pain: algogenic stimuli projecting grossly towards subcortical, limbic or even encephalic trunk, conform a sort of pain devoid of bioethical concerns. Clinical reports of malformations and/or brain pathologies in newborns and adults show that attitudes, facial expressions and postures are related with sensation of pain. It is necessary to distinguish between the presence and functioning of a complex neurocircuit for conscious neural processing and the ability to feel pain. Also, brainstem contains several structures that are likely involved in the generation and experience of pain. ${ }^{2}$

\section{Conclusions}

Thus, in mammals pain feeling is generated in the cortex while in other animal taxa it is generated in different brain areas. These regions are functionally analogous to the segmental neurozone, to perform a similar function with evoked
Correspondence:Michele Panzera, Laboratory of Veterinary Ethology, Department of Veterinary Science, University of Messina, Messina, Italy.

E-mail:mpanzera@unime.it

Key words: Animal; pain; sentience.

Conference presentation: this paper was presented at the Second Centro 3R Annual Meeting - 3Rs in Italian Universities, 2019, June 20-21, University of Genoa, Italy.

Received for publication: 28 October 2019. Accepted for publication: 6 November 2019.

This work is licensed under a Creative Commons Attribution NonCommercial 4.0 License (CC BY-NC 4.0).

(C) Copyright: the Author(s), 2019

Licensee PAGEPress, Italy

Biomedical Science and Engineering 2019; 3(s2):99 doi:10.4081/bse.2019.99

features and neuromodulatory agents that conform the nociceptive stimulus. It is not only the cortex that counts! In lights of these considerations it is reasonable to believe that the appreciation of these different perceptions deserves bioethical consideration.

\section{References}

1. Key B. Why fish do not feel pain. Animal Sentience 2016;3.

2. Petrovic P, Petersson KM, Hansson P, Ingvar M. Brainstem involvement in the initial response to pain. Neuroimage 2004;22:995-1005. 